\title{
Conservative, Dissipative and Super-diffusive Behavior of a Particle Propelled in a Regular Flow
}

\author{
Gil Ariel* $\quad$ Jeremy Schiff $^{\dagger}$
}

November 14, 2019

\begin{abstract}
A recent model of Ariel et al. [1] for explaining the observation of Lévy walks in swarming bacteria suggests that self-propelled, elongated particles in a periodic array of regular vortices perform a super-diffusion that is consistent with Lévy walks. The equations of motion, which are reversible in time but not volume preserving, demonstrate a new route to Lévy walking in chaotic systems. Here, the dynamics of the model is studied both analytically and numerically. It is shown that the apparent super-diffusion is due to "sticking" of trajectories to elliptic islands, regions of quasi-periodic orbits reminiscent of those seen in conservative systems. However, for certain parameter values, these islands coexist with asymptotically stable periodic trajectories, causing dissipative behavior on very long time scales.
\end{abstract}

\section{Introduction}

Lévy walks are random process that are characterized by trajectories that have straight stretches for extended lengths whose variance is infinite. It is well known that Lévy walks can arise in chaotic systems [2]. Indeed, the literature contains a potpourri of models and theories explaining the mechanisms underlying such dynamics. Notable examples include Hamiltonian systems [3, 4, 5, 6], area-preserving maps [7, 8, 9], chaotic or random velocity fields [10], time varying fields in resonance [11, 12, 13, dissipative systems [14, turbulence [15] and more [16].

Here, we consider the following set of ordinary differential equations (ODEs), describing the position and orientation of an infinitesimal, self-propelled, prolate spheroid that is advected by an array of vortices:

$$
\begin{aligned}
& \dot{x}=\sin \pi x \cos \pi y+V \cos \pi z, \\
& \dot{y}=-\cos \pi x \sin \pi y+V \sin \pi z, \\
& \dot{z}=\sin \pi x \sin \pi y-2 D \cos \pi x \cos \pi y \cos \pi z \sin \pi z .
\end{aligned}
$$

Here $x$ and $y$ describe the position of a point particle moving in the plane and advected along an array of vortices with flow lines $\psi(x, y)=\pi^{-1} \sin \pi x \sin \pi y$. Assuming Stokes dynamics, the particle pushes itself at constant speed $V \in[0,1]$ in the direction of its "head" with

*Department of Mathematics, Bar-Ilan University, ramat-gan 5290002, Israel. (arielg@math.biu.ac.il)

${ }^{\dagger}$ Department of Mathematics, Bar-Ilan University, ramat-gan 5290002 Israel. (schiff@math.biu.ac.il) 

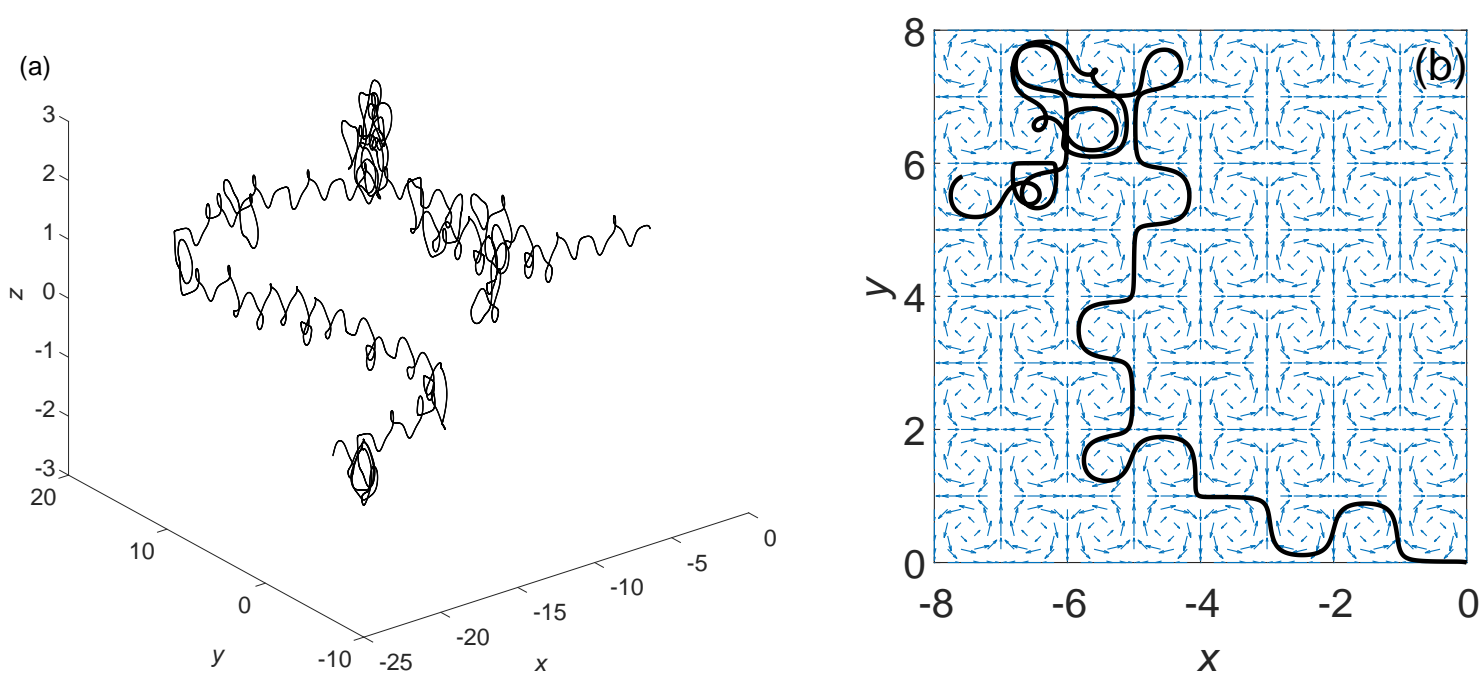

Figure 1: (a) An example trajectory, with $V=1 / 2, D=12 / 13$ and initial conditions $(x(0), y(0), z(0))=(0,0,0.9)$. (b) Projection of part of the trajectory onto the $x$ - $y$ plane, with the vortex field displayed.

angle $\pi z$ relative to the $x$-axis. The rotation of the head is given by Jeffery's equation, where $D \in[0,1]$ is a shape parameter. Figure 1 shows (a) a typical trajectory and (b) projection of part of the trajectory onto the $x$ - $y$ plane, on which the vortex field is displayed. The ODEs (1.1) have been considered by Torney and Neufeld [17] and independently by Ariel et al. [1] as a simplified model for explaining the observation of Lévy walks in swarming bacteria [18]. In the following we focus on the dynamics of (1.1) in order to better understand the mechanisms resulting in super-diffusive trajectories.

This paper is organized as follows. In section 2 we go through the basic analytic properties of the system (1.1) and in particular its symmetries. The system has a time reversal symmetry, which has important implications for the dynamics. We discuss periodic orbits, and give a numeric-aided proof of the existence of a periodic orbit in a specific case. We show this orbit is surrounded by an elliptic island, similar to those observed in conservative systems. The "stickiness" of such islands (i.e. the fact that nearby orbits can get stuck close to the island for long periods of time) seems to be a significant mechanism underlying super-diffusivity. In section 3 numeric results are presented. A specialized numerical method is described that gives accurate solutions over extended time scales. This is used to investigate the dependence on the parameters $D$ and $V$ of various properties of the system, such as the exponent of super-diffusivity. Rather surprisingly, a region of parameter space is identified in which the numerics indicate that this exponent has the constant value 2, corresponding to ballistic motion (also called accelerator modes [19]). Further exploration shows that in this region there exist attractive periodic orbits, and suggests that for some parameter values, almost all orbits outside the elliptic islands tend to these orbits, but only after very long times. For other parameter values, however, only orbits sufficiently close to the attractive orbits are attracted to them. We conclude in Section 4. 


\section{Basic analysis}

First we note that the system is not Hamiltonian, as the number of variables is odd. Indeed the dynamics is not even volume preserving, as the divergence of the right hand side of (1.1) is $-2 \pi D \cos \pi x \cos \pi y \cos 2 \pi z$, which is typically not zero.

Next, it is easily verified that the ODE system (1.1) is invariant under the symmetry $(x, y, z) \mapsto(x+2, y+2, z+2)$. Thus the dynamics can be thought of as occurring in the 3 -dimensional torus $\Omega=[-1,1]^{3}$. Additional symmetries are

- $(x, y, z) \mapsto(-x, y, 1-z)$

- $(x, y, z) \mapsto(x,-y,-z)$

- $(x, y, z) \mapsto(x+1, y+1, z)$

and compositions of the above. Hence the basic domain $\Omega$ is further partitioned into 8 symmetric parts.

Most importantly, the dynamics is reversible, in the sense that there exists an involution $G$ of $\Omega$ (i.e. a map from $\Omega$ to itself with $G^{2}=I$ ) such that solving the ODE backward in time is the same as applying $G$, solving forward in time, and then applying $G$ again. If we denote the propagator of (1.1) as $\Phi_{t}$ (i.e $\Phi_{t}\left(x_{0}, y_{0}, z_{0}\right)$ is the solution of (1.1) with initial condition $\left.\left(x_{0}, y_{0}, z_{0}\right)\right)$, then the dynamics is reversible if $\Phi_{t} G \Phi_{t} G=1$. It is easily verified that taking

$$
G:(x, y, z) \mapsto\left(y, x, \frac{3}{2}-z\right)
$$

satisfies this requirement. Reversible dynamical systems can exhibit types of behavior associated with conservative systems, specifically they can have "elliptic islands" in which a periodic elliptic orbit is surrounded by invariant surfaces, between which there may be other periodic orbits, surrounded by other invariant surfaces, and so on. However, they can also exhibit features of dissipative systems, such as attractors and repellors. See, for example, the review by Roberts and Quispel [20], for the case of reversible maps of the plane, and the two more recent works [21, 22]. In a reversible system, fixed points and periodic solutions can be either symmetric (i.e. mapped to themselves by the involution $G$ ) or asymmetric. Clearly, attractors and repellors cannot be symmetric, as $G$ must map an attractor to a repellor and vice-versa.

The first step in the investigation of the dynamics of any system is to find the fixed points, and try to spot simple invariant manifolds. For (1.1), it is straightforward to check that there are 16 fixed points, two of these being the points with $y=z=0, \sin \pi x=-V$, and the remaining 14 being generated from these by application of the three symmetries and the time reversing involution $G$ described above. Looking at the linearizations near the 16 fixed points, 8 have two eigenvalues with positive real part and one with negative real part, and the remaining 8 have two eigenvalues with negative real part and one with positive real part.

The eigenvalues are all real if $\left(1+\left(D-\frac{1}{2}\right)^{2}\right)\left(1-V^{2}\right) \geq 1$, otherwise there is a complex conjugate pair. With regard to invariant manifolds, the circle $y=z=0$ is invariant, as are 7 other circles obtained by the applying the symmetries and $G$. Each of these circles passes through two of the fixed points (one with a two-dimensional stable manifold, one with a twodimensional unstable manifold), and, apart from these points, consists of a pair of heteroclinic orbits. 

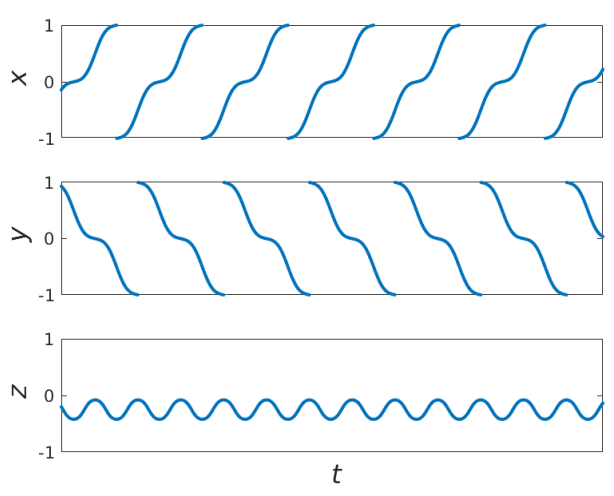

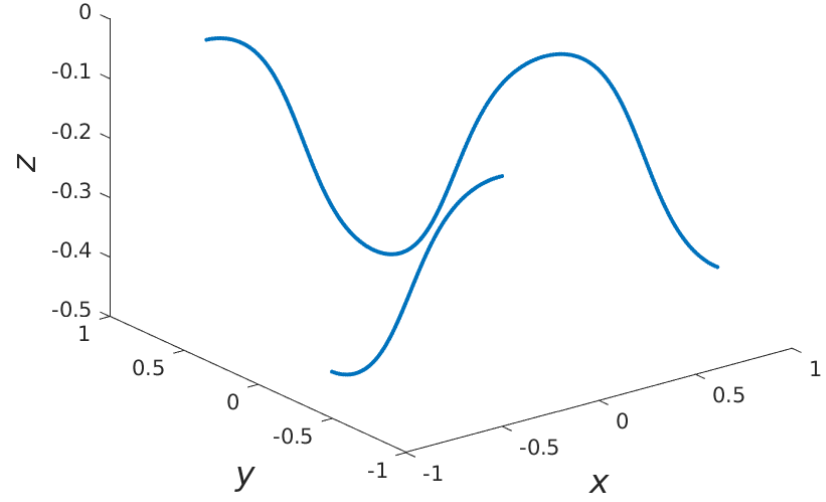

Figure 2: A periodic solution in the case $D=12 / 13, V=1 / 2$, passing approximately through the point $(1.858624,0.930363,-0.2)$. Left: $x, y, z$ as functions of $t$. Right: 3 -d view of the orbit.

The next question to consider is whether there exist periodic orbits. There is, in general, no analytic way to resolve this, but numerical experiments show that the system (1.1) does indeed have many periodic orbits. Note that for a general flow $\dot{\mathbf{x}}=F(\mathbf{x})$ on the torus $[-1,1]^{d}$ a periodic orbit takes the form

$$
\mathbf{x}(t)=\frac{2 \mathbf{n} t}{T}+\mathbf{y}(t)
$$

where $\mathbf{y}: \mathbf{R} \rightarrow \mathbf{R}^{d}$ is periodic with period $T$ and $\mathbf{n} \in \mathbf{Z}^{d}$. Thus, for the system (1.1), each periodic orbit is characterized by 3 integers, which specify the number of times the orbit winds around the torus in each of the $x, y, z$ directions in the course of a single period. In the following we take parameter values $D=12 / 13$ and $V=1 / 2$, which are values that are realistic for the spreading phenomenon of bacteria within a swarm [1]. For these parameters one periodic orbit goes approximately through the point $\left(x_{p}, y_{p}, z_{p}\right)=(1.858624,0.930363,-0.2)$. This has period approximately 2.77 and the winding numbers are $n_{x}=1, n_{y}=-1, n_{z}=0$. See figure 2. This orbit is invariant under $G$ (Note that $G$ changes the sign of both $n_{x}$ and $n_{y}$ while leaving $n_{z}$ invariant, thus a necessary condition for $G$ invariance is $n_{x}=-n_{y}$ ). The orbit is also invariant under one of the 3 symmetries of the system, but applying the other two symmetries generates 3 more periodic orbits, with winding numbers (1) $n_{x}=n_{y}=1, n_{z}=0$, (2) $n_{x}=n_{y}=-1, n_{z}=0$ and (3) $n_{x}=-1, n_{y}=1, n_{z}=0$.

The periodic orbit presented in the previous paragraph was found by looking at the return map to a surface of section, and we also use this to give a numeric-aided proof of existence of the orbit and, in the next paragraph, to study its stability. Specifically, we use the surface of section $z=-0.2$. We see that the orbit hits this 4 times in a period, once in each quadrant of the $x, y$-plane, so we restrict to the quadrant $-1<x<0,0<y<1$. Denote by $(F(x, y), G(x, y))$ the first return map to this section, starting from the point $(x, y)$. Thus we seek a solution of

$$
\begin{aligned}
& x=F(x, y) \\
& y=G(x, y) .
\end{aligned}
$$

Consider a square domain $A$ on the surface of section in which the first return time is bounded by a constant $\tau$. Denote the RHS of (1.1) as $H(x, y, z)$. Then, the derivatives of $F$ and $G$ are 


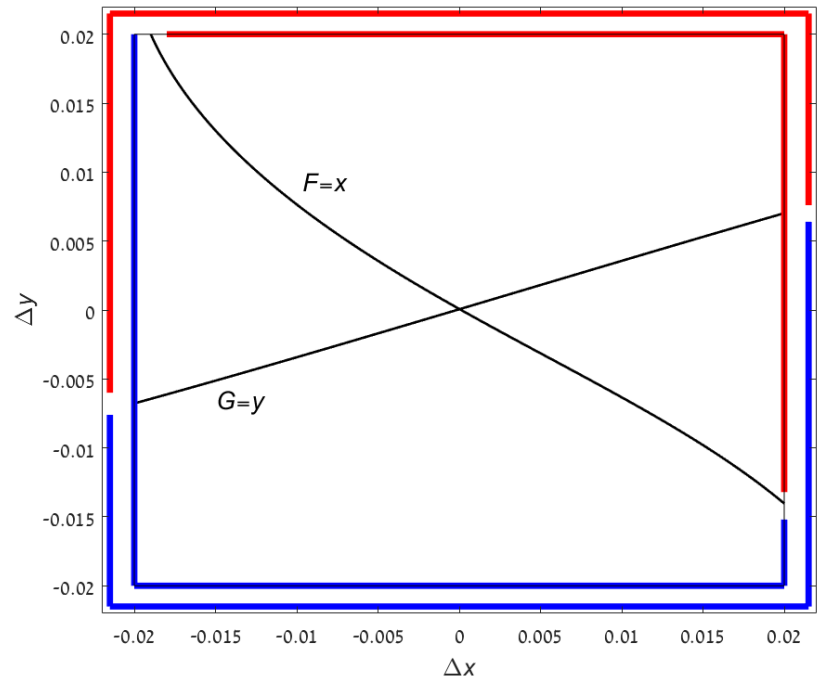

Figure 3: Proof of existence of periodic solutions. Outer square blue: $F(x, y)-x$ is positive. Outer square red: $F(x, y)-x$ is negative. Inner square blue: $G(x, y)-y$ is positive. Inner square red: $G(x, y)-y$ is negative. The zero level set of $F-x$ connects some point in the gap on the top of the outer square with some point in the gap on the right side of the outer square. The zero level set of $G-y$ connects some point in the gap on the left side of the inner square with some point in the gap on the right side of the inner square. Clearly they must intersect in the interior.

bounded by $\tau \max _{A}|\nabla H|$. The accuracy of a numerical computation of $F$ and $G$ with a given integration scheme also depends on similar bounds. The $n$ 'th order partial derivatives of $H$ are trivially bounded in the sup norm by $2(2 \pi)^{n}$. Hence, we can obtain strict bounds for both the accuracy of our numerical computation of $F$ and $G$ and their derivatives. Let tol $>0$ denote a given accuracy tolerance. We compute $F$ and $G$ on a uniform grid along the boundary of $A$ and note all points in which we can guarantee that $F(x, y)-x$ and $G(x, y)-y$ are strictly positive or negative (i.e. larger than tol or smaller than -tol). The grid spacing should be small enough such that $F$ and $G$ cannot change by more than tol between neighboring points. Figure 3 depicts our results with $A=\left[x_{p}-0.02, x_{p}+0.02\right] \times\left[y_{p}-0.02, y_{p}+0.02\right]$ and tol $=10^{-3}$. The plot is shifted so that the fixed point is at the origin. Blue (red) lines show positive (negative) values of $F-x$ (outer square) and $G-y$ (inner square). Segments along the boundary of $A$ which are not colored cannot be classified as positive or negative within the given precision. As the figure shows, the region in which $F-x$ changes sign does not overlap that of $G-y$. Furthermore, following the boundary of $A$ in a fixed orientation (e.g., clock-wise), the regions in which $F-x$ and $G-y$ change sign alternate. It follows that the zero level curve of $F-x$ must intersect that of $G-y$ inside the square. This intersection point is a fixed point for the return map and hence a periodic solution of (1.1).

The stability of periodic orbits can be studied by numerically plotting the return map to the surface of section $z=-0.2$ near one of the periodic orbits, see Figure 4(a). Each color corresponds to a different initial condition. The concentric ellipsoid-like curves are invariant curves corresponding to quasiperiodic solutions, and their existence shows that the periodic 
solution corresponding to the fixed point in the center (which we call the T1 solution) is neutral (i.e. stable but not asymptotically stable). Further away from the center some of the invariant curves break up. Chaotic dynamics can be observed, interspersed with additional islands of stability around periodic orbits of longer period. Specifically in figure 4(a) we see five islands, surrounding an orbit we call T5, with period approximately 5 times that of T1. Zooming in (see figures 4(b)-(d)), additional islands of stability can be seen, identified with increasingly finer structures and longer periods. We identify periods up to 1050 times the period of T1. At the center of the large island in figure 4(d) there is a hyperbolic fixed point. This phenomenon will be explained in section 3.3 .
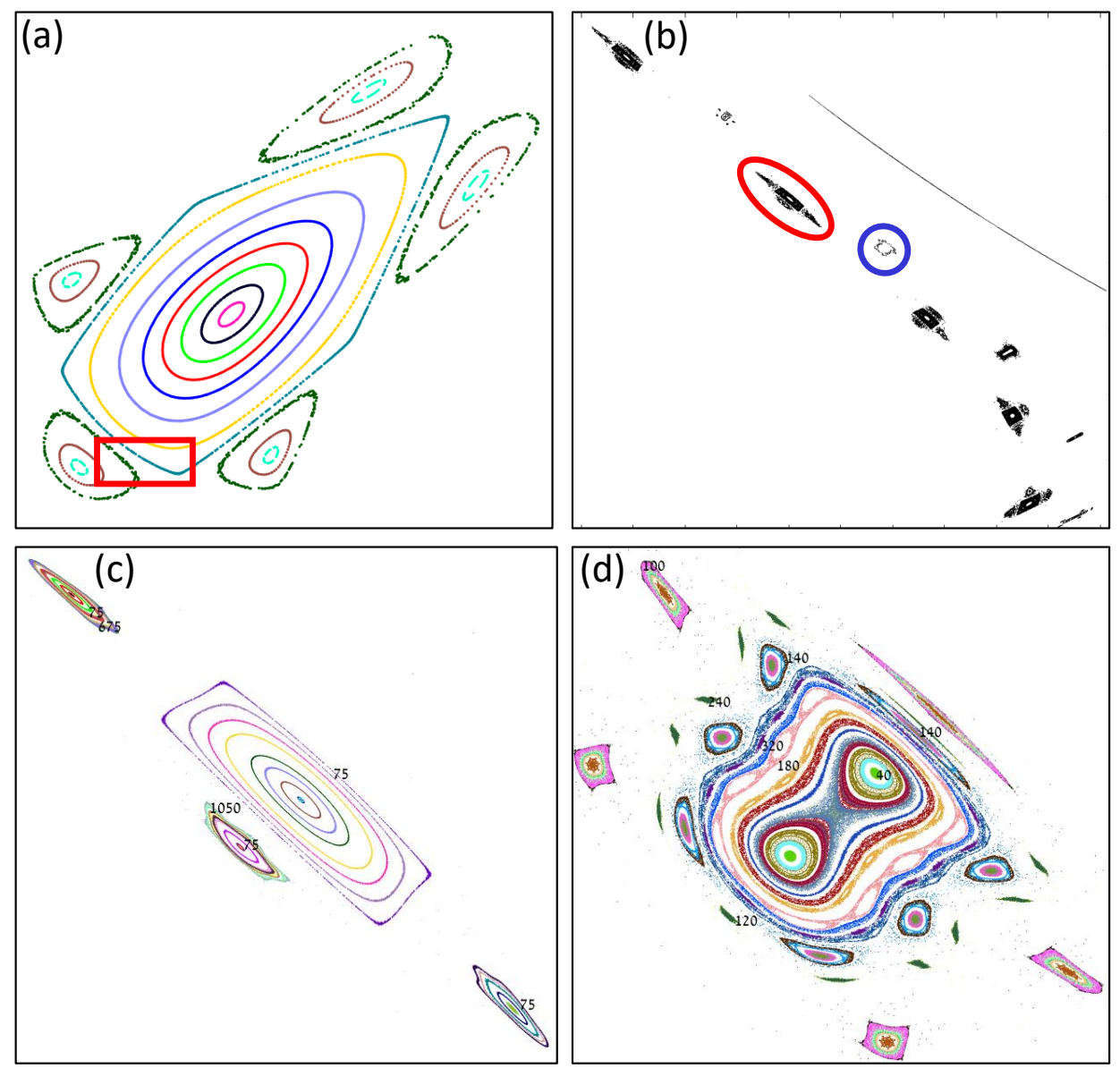

Figure 4: Periodic orbits and their stability. All figures show the return map to the surface of section $z=-0.2$. Each color corresponds to a different initial condition. (a) The region $[1.75,2] \times[0.85,1.05]$ showing the T1 and T5 orbits. (b) A zoom into the red rectangle in (a) reveals additional features. (c) A zoom into the region $[1.8108,1.8132] \times[0.8831,0.8848]$, including the red ellipse in (b). Numbers denote the period multiplier. (d) A zoom into the region $[1.8092,1.80995] \times[0.8862,0.88673]$, including the blue circle in $(b)$. 
Thus we see that the system has neutrally stable periodic orbits, and other solutions that remain close to, but are not attracted to, the periodic orbits. How do generic orbits behave? In figure 5 we show, for $D=12 / 13, V=1 / 2$, all points on the return map to the surface of section $z=-0.2$ for 100 orbits, run from $t=0$ until $t=2000$. The four ghost-like "holes" correspond to the regions of the islands of stability, one of which is described in figure 4. None of the 100 orbits were taken within these regions, which occupy approximately $1 / 50$ of the total area. However, we note that a large fraction of the points are found in certain regions that, roughly speaking, surround the holes. It is well known that trajectories can "stick" to the area close to regular regions [20]. When the sticking time has a power-law distribution, then super-diffusion and Lévy walks can occur. Loosely speaking, the reason is that while a trajectory is close to a periodic or quasi-periodic orbit, it appears ballistic (in the unwrapped space). The result is a Lévy walk [2]. The route from chaos to Lévy walks has been mainly studied in the context of Hamiltonian or area-preserving systems [7, 8, 23]. Sticking has been attributed to long escape times from regions that are bounded by broken KAM trajectories [23, 24]. To the best of our knowledge, the origin of super-diffusion in the more general class of reversible systems is not well understood [20].

We note that in addition to the general accumulation of density in some regions close to the "holes", the plot in figure 5 displays much additional structure, both in and away from the regions of high density. In the sequel, we refer to the region of phase space outside the islands of stability as the chaotic region

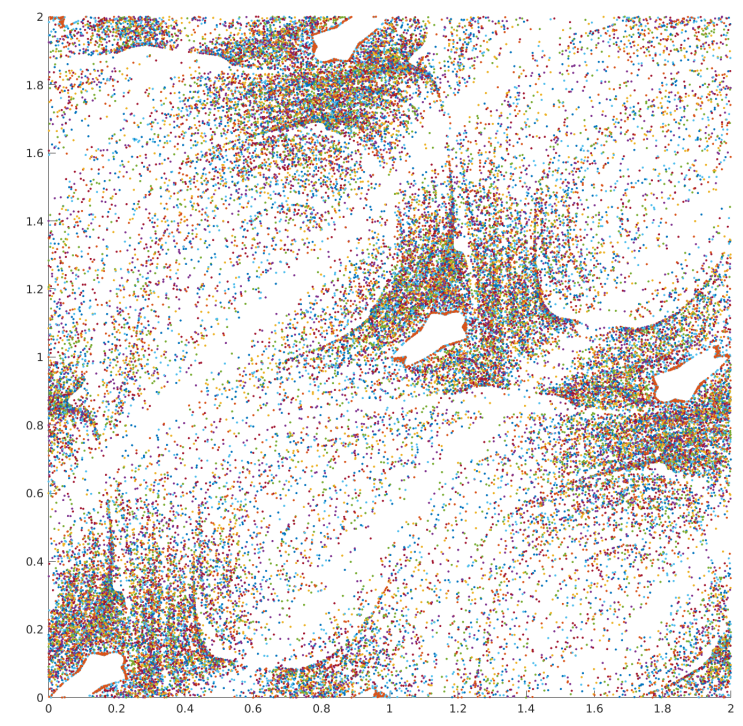

Figure 5: Points on the return map to the surface of section $z=-0.2$ for 100 orbits, run from $t=0$ until $t=2000$, for $D=12 / 13, V=1 / 2$. The holes correspond to the regions of the islands of stability, which are "sticky" and have regions of high density close to them, corresponding to solutions that are close to ballistic for long periods of time. 


\section{Long-time Numerics}

\subsection{A bespoke numerical method}

Estimation of the statistical properties of trajectories, for example the mean square displacement, with reasonable accuracy, requires numerical integration of (1.1) for long times. Standard numerical methods, e.g. Runge-Kutta, are notoriously inappropriate for integrating chaotic systems, and adaptive methods can become prohibitively inefficient. The problem is worse for long-time integration of equations such as (1.1). Small noise due to numerical errors can make a trajectory jump from outside to inside one of the quasi-periodic regions described above, or vice-versa. This leads to a qualitatively different geometry of trajectories.

The antidote to these problems is to use an integrator that preserves the geometric properties of the system. Integrators that preserve symmetries, including time reversal symmetries, have been developed, for example in [25. We take another approach. Elliptic islands are more commonly seen in Hamiltonian and volume-preserving systems. We show how the system (1.1) can be embedded (as motion on a 3-d invariant submanifold) in a 4-d volume preserving system, and apply a volume-preserving integrator to this. Although we find that it is necessary to project back the relevant 3-d submanifold, we hope this method retains sufficient geometric structure to accurately integrate the system.

Consider the 4-d system

$$
\begin{aligned}
& \dot{w}=-\sin \pi x \sin \pi y+D \cos \pi x \cos \pi y \sin 2 \pi z, \\
& \dot{x}=\sin \pi x \cos \pi y+V \cos \pi w, \\
& \dot{y}=-\cos \pi x \sin \pi y+V \sin \pi z, \\
& \dot{z}=\sin \pi x \sin \pi y+D \cos \pi x \cos \pi y \sin 2 \pi w .
\end{aligned}
$$

This is obtained from (1.1) by appending the equation for $w$ and replacing $z$ by $-w$ in two places. The RHS of this new system is now divergence free, and thus it is a volume preserving system. It is also straightforward to check that the surface $w=-z$ is invariant, and reduction to this surface gives the original system.

Following an idea of Feng Kang [26], we find three Hamiltonians such that the dynamics is a composition of three Hamiltonian maps. There is some freedom in choosing how to split

the system. Here, since the convection of particles along the flow lines is divergence free, we keep $x$ and $y$ as a pair. Similarly, for symmetry reasons, we pair $x$ with $w$ and $y$ with $z$. Overall, we seek three Hamiltonians, $H_{x y}, H_{w x}$ and $H_{z y}$ such that

$$
\begin{gathered}
\dot{x}=\frac{\partial H_{x y}}{\partial y}+\frac{\partial H_{w x}}{\partial w}, \quad \dot{y}=-\frac{\partial H_{x y}}{\partial x}+\frac{\partial H_{z y}}{\partial z} \\
\dot{w}=-\frac{\partial H_{w x}}{\partial x}, \quad \dot{z}=-\frac{\partial H_{z y}}{\partial y}
\end{gathered}
$$

We find that a convenient choice is given by,

$$
\begin{aligned}
H_{x y} & =\pi^{-1} \sin \pi x \sin \pi y \\
H_{w x} & =\pi^{-1}[V \sin \pi w-\cos \pi x \sin \pi y-D \sin \pi x \cos \pi y \sin 2 \pi z], \\
H_{z y} & =-\pi^{-1}[V \cos \pi z+\sin \pi x \cos \pi y-D \cos \pi x \sin \pi y \sin 2 \pi w] .
\end{aligned}
$$


Denote by $N_{x y}^{h}, N_{w x}^{h}$ and $N_{z y}^{h}$ a numerical integration scheme with step size $h$ for each of the Hamiltonian systems respectively. Then, integration of $(3.2)$ can be performed by a symmetric splitting method,

$$
N_{z y}^{h / 2} N_{w x}^{h / 2} N_{x y}^{h} N_{w x}^{h / 2} N_{z y}^{h / 2} .
$$

In practice, we apply a Verlet method for each of the integrators. Since the Hamiltonians are not separable, the Verlet schemes are implicit. However, the implicit equation in each step is scalar and is easily solved to machine precision with a few Newton-Raphson iterations. In order to make sure that the solution is consistent with the original system (1.1), we project the solution onto the $w=-z$ submanifold after each integration step, using the simple linear projection $z \leftarrow(z-w) / 2, w \leftarrow(w-z) / 2$. Although the projection is not reversible, in practice, using this scheme with a step size of $h=10^{-3}$ or smaller is accurate to a high degree of precision.

\subsection{Super diffusive properties}

The parameters $D$ and $V$ determine the geometric properties of trajectories. One simple way to quantify how super-diffusive trajectories are is by calculating the Mean Squared Displacement (MSD). The physical motivation of the model is in describing transport or diffusion of particles in a flow. Accordingly, we focus on diffusion in the unwrapped $x$ - $y$ plane, i.e., in $\mathbb{R}^{2}$ and not on the torus. Denoting by $r(t)=(x(t), y(t))$ the position in the $x$-y plane at time $t$, the MSD is defined as

$$
\operatorname{MSD}(t)=<|r(t+s)-r(s)|^{2}>\text {. }
$$

where $\langle\cdot\rangle$ denotes a suitable average. This might be an average over all times $s$ for a specific orbit (in the chaotic region), or an average over all possible initial conditions $r(s)$ for some fixed $s$. Typically,

$$
\operatorname{MSD}(t) \sim D t^{\alpha}
$$

where $0 \leq \alpha \leq 2$. $\alpha$ is called the exponent of diffusivity. Bounded trajectories have $\alpha=0$. In cases where the standard central limit theorem holds, we have regular diffusion with $\alpha=1$. $\alpha=2$ implies ballistic motion with an effectively constant velocity. Systems with $0<\alpha<1$ are called sub-diffusive and systems with $1<\alpha<2$ are called super-diffusive. Figure 6 shows the numerically calculated MSD exponent $\alpha$ as a function of $D$ and $V$. Values of $D$ and $V$ were taken in increments of 0.01 . For each value of $D$ and $V$ a single orbit of length $10^{4}$ was computed, and the MSD calculated, as an average along the orbit, for a number of values of $t$, allowing the exponent of super-diffusivity to be found by a linear fit of values of $\log \operatorname{MSD}(t)$ as a function of $\log t$.

One conclusion that can be drawn from this plot is that in certain regions of parameter space we have $\alpha=2$, i.e. the dynamics appears ballistic. This turns out to be a result of the existence of asymptotically stable periodic orbits, which will be explored further in the next subsection. The results are arguably consistent with the hypothesis that for $0<D, V<1$ we always have $\alpha>1$, i.e., trajectories are super-diffusive. However, the number of values of $\alpha$ observed above 1.4 is small, and there is sensitive dependence on the values of $V$ and $D$, so it is not impossible that in fact $\alpha=1$ or $\alpha=2$ for all values of $V$ and $D$, c.f. the discussion in [19] and [27]. It is evident that an orbit of length $10^{4}$ is not long enough to reliably compute the average defining the MSD. However, it is also clear that on time scales of this length, there is significant evidence of super-diffusive behavior. 


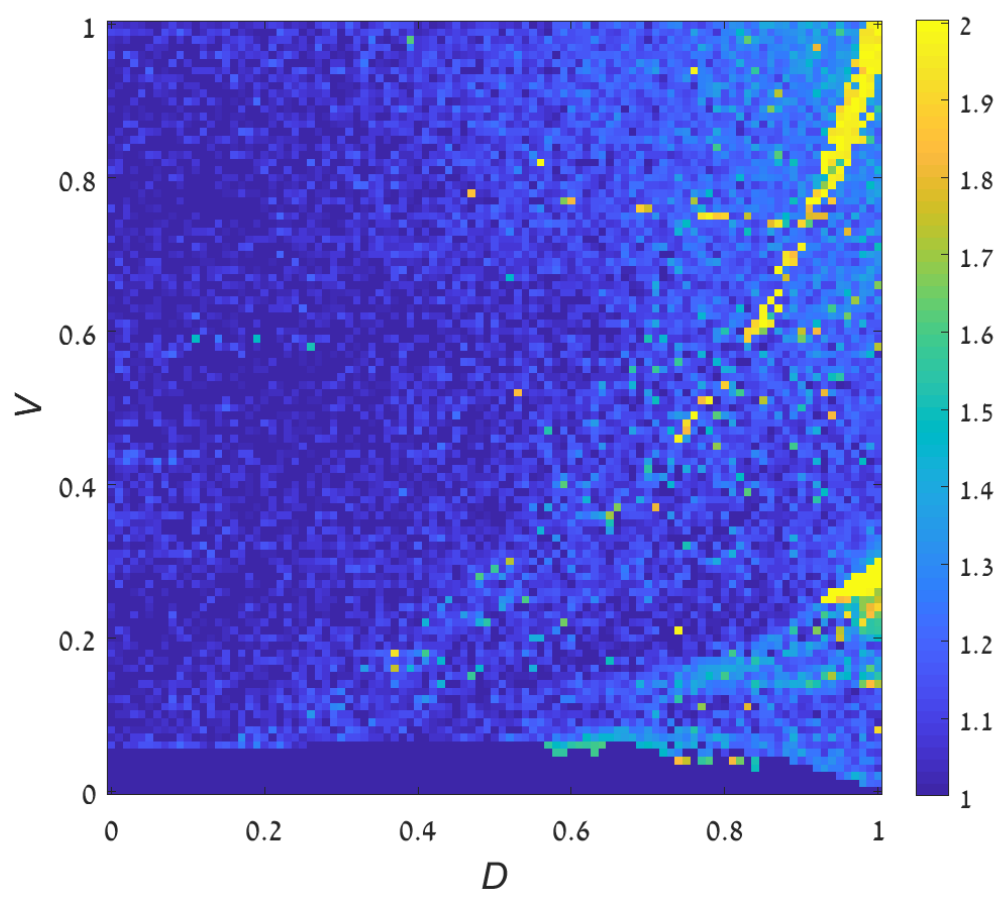

Figure 6: Measured exponent of super-diffusivity as a function of $D$ and $V$

\subsection{Dissipative behavior}

As mentioned in the previous section, in Figure 6 we see regions in which the exponent of super-diffusivity appears to be 2 . Further numerical investigation of these regions shows that the reason for this is that in these regions, there exist asymptotically stable periodic orbits, and, furthermore, generic orbits outside the elliptic islands eventually tend to one of these attractors. As we have noted previously, the divergence of the right hand side of 1.1) is $-2 \pi D \cos \pi x \cos \pi y \cos 2 \pi z$, which is not of fixed sign. If generic orbits tend to an attractor, we expect the time average of the divergence to be negative. In Figure 7 we show the dependence on $D$ and $V$ of the time average of the divergence along a single orbit of length $10^{4}$ in the chaotic region of the flow. As expected, we see regions in which the time average of the divergence is significantly negative.

How do the attractors appear/disappear at the edges of the regions in parameter space in which they occur? We studied the change in the attractor that exists for $V=0.6, D=0.84$ as $D$ is changed for fixed $V$. As $D$ is reduced (around $D=0.83$ ) there is a period doubling bifurcation, which initiates a period doubling cascade as $D$ is reduced further, leading to the disappearance of the periodic orbit. As $D$ is increased, at some stage (before $D=0.86$ ) the attractor loses the property of being a global attractor for generic orbits outside the elliptic islands. Instead, the attractor is surrounded by an invariant surface, which is the boundary of its basin of attraction, and this is surrounded by other invariant surfaces. In figure 8 we show part of the return map to the surface of section $\theta=0.75$ for parameter values $V=0.6$ and $D=0.865$. A total of 7 orbits are shown. For 5 of these, the orbits fill a curve. For the remaining 2 , the orbits tend to a fixed point at (approximately) $(1.15188,0.32300)$. As $D$ 


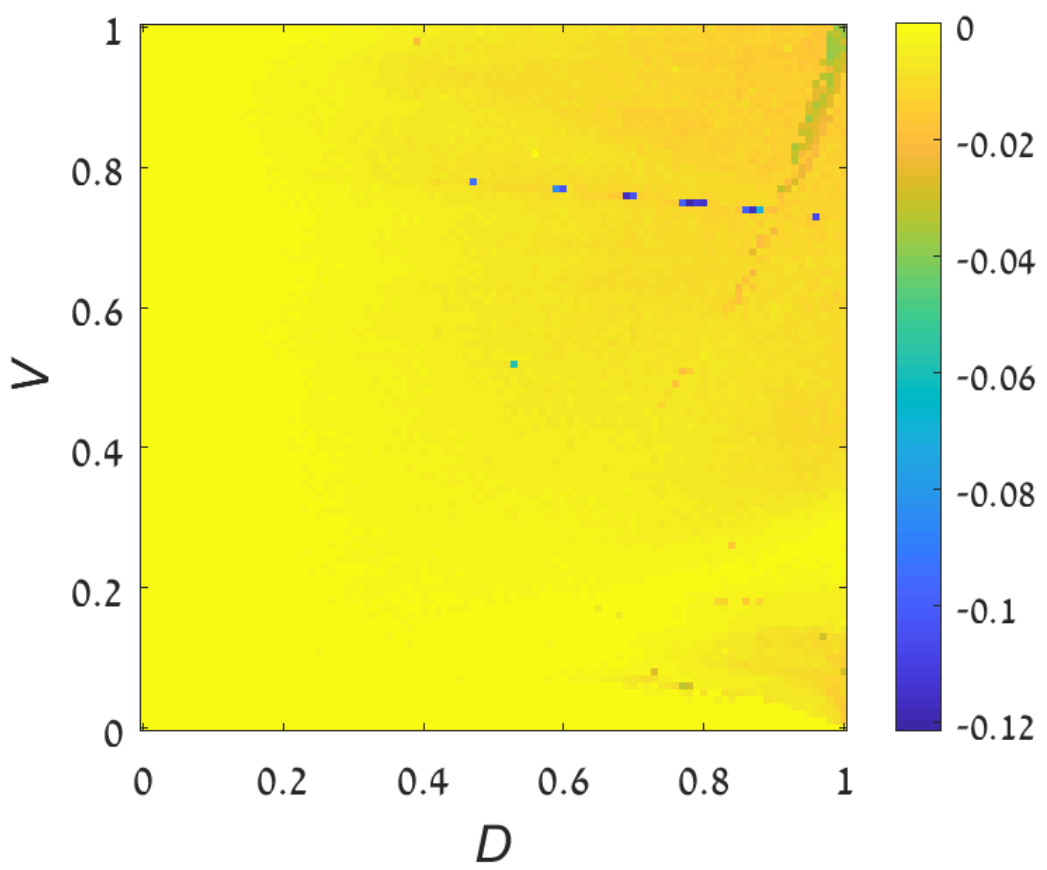

Figure 7: Time average of the divergence of the flow along an orbit of length $10^{4}$ in the chaotic region of flow, as a function of $D$ and $V$.

is increased even further, this fixed point changes from being asymptotically stable to being neutrally stable, and an elliptic island appears (for the return map, the two eigenvalues go from being both real and smaller than 1 in absolute value, to being a complex conjugate pair of absolute value 1). Approaching the bifurcation point, the area of the basin of attraction of the stable orbit seems to shrink to zero.

Section 5.2 of [20] describes the bifurcation of a neutrally stable symmetric fixed point in a reversible map of the plane to a hyperbolic fixed point and a symmetric, neutrally stable two-cycle. The observation is made that some of the "outer" invariant curves surrounding the original fixed point persist, and surround the hyperbolic fixed point and the two-cycle after the bifurcation. This is the situation observed in Figure 4(d). The hyperbolic fixed point in this figure (between the two sub-islands of the big central island) has one eigenvalue with absolute value less than 1 , and the other with absolute value greater than 1 . The situation we see in figure 8, however, is formed (as $D$ decreases, for fixed $V$ ) by the bifurcation of a neutrally stable asymmetric fixed point to a hyperbolic stable fixed point, with two eigenvalues with absolute value less than 1. Recall that in a reversible map, all symmetric fixed points are necessarily neutrally stable; but there can also exist asymmetric neutrally stable fixed points. Also in the case of this bifurcation, some of the invariant curves surrounding the fixed point persist, as the type of the fixed point changes, giving rise to the picture in figure 8 . We are not aware that the occurrence of such a bifurcation has previously been reported.

As a final note on this section of dissipative behavior, we mention that even in the case that all orbits outside outside the elliptic islands tend to an attractor, resulting, ultimately, in ballistic motion, the typical time scale on which this happens is very long (of order $10^{5}$ in 


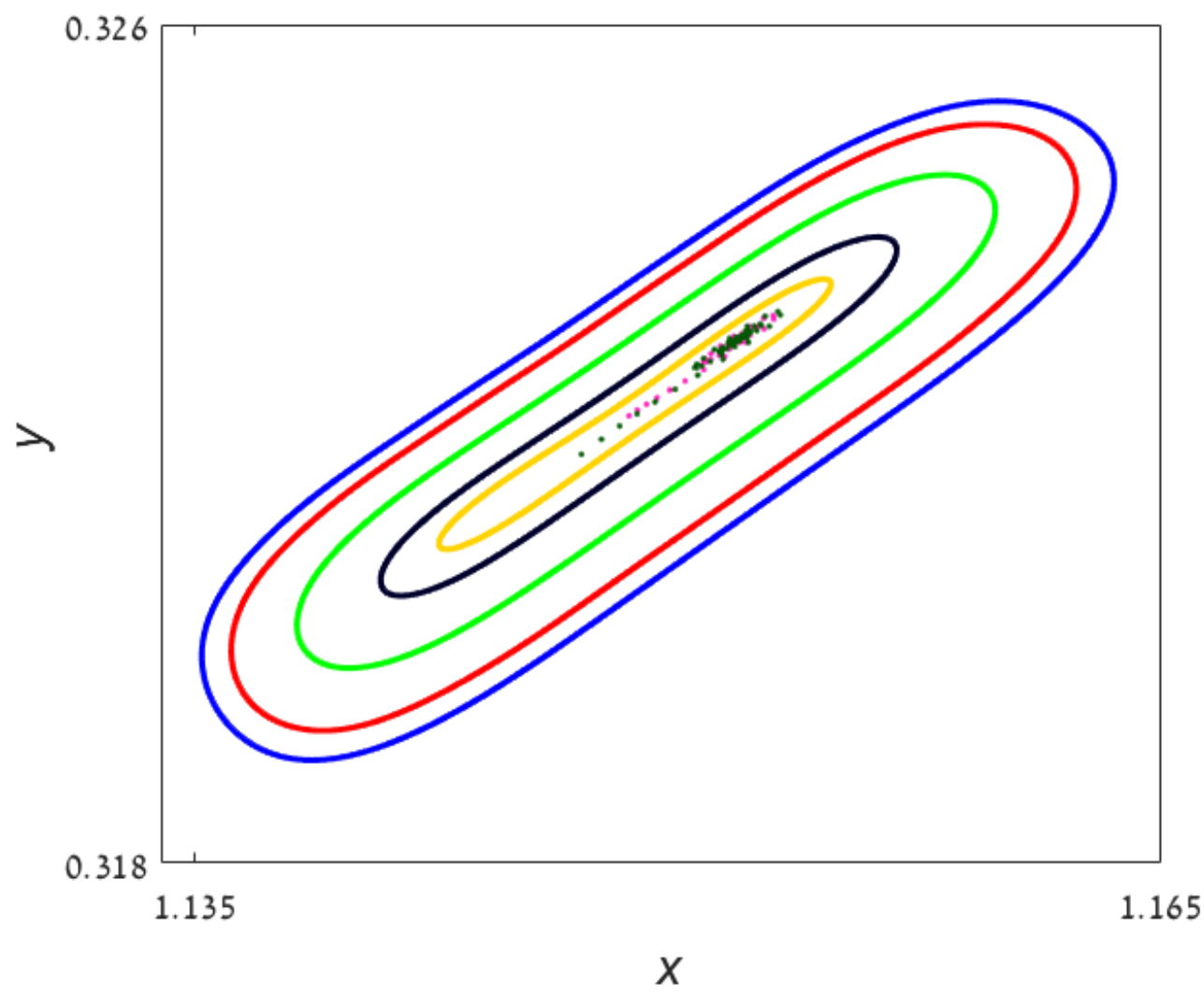

Figure 8: Return map to $\theta=0.75$ for $V=0.6, D=0.865$. An asymptotically stable fixed point sits inside a family of invariant curves. The innermost curve bounds the basin of attraction of the fixed point.

cases we have investigated). On shorter time scales the phenomenon of stickiness around the elliptic islands dominates.

\subsection{Sticking times}

We report one final numerical study, on the distribution of sticking times. In the case that there are no attractors (and in the case that these have bounded basins of attraction), we have argued that the dominant feature of the dynamics outside the elliptic islands is that orbits spend a long time "sticking" to the islands. It is not clear precisely how to quantify this. We performed the following experiment: In the case $D=12 / 13, V=1 / 2$ we looked at a single very long orbit in the chaotic regime. We chose a rectangular region around the elliptic islands in the Poincare section $z=-0.2$, i.e. the holes in figure 5 . We recorded the lengths of the times that the orbit stayed in this region, i.e. the "sticking time" and used these to build an empirical cumulative distribution function $F(t)$, that gives the probability that a given sticking time is less than $t$. We expect power law behavior for the tail of this distribution, i.e.

$$
1-F(t) \sim C t^{-\gamma}, \quad t \rightarrow \infty
$$



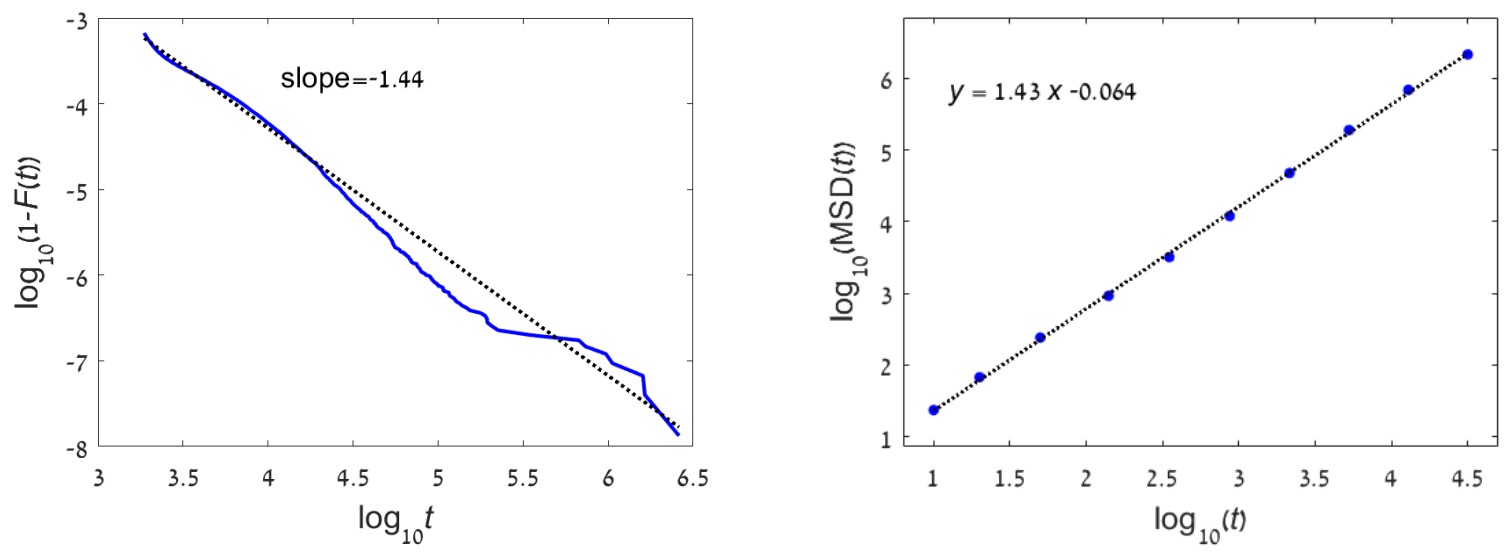

Figure 9: Left: The cumulative distribution function of sticking times around the regular islands has approximately a power-law tail with exponent $\gamma=1.44$. Right: The MSD of the same orbit $\left(T=8 \cdot 10^{7}\right)$ shows super diffusion with exponent $\alpha \approx 1.43$. The result is consistent with the standard Lévy walk model, in which $\gamma=3-\alpha$ [16].

for some positive constants $C$ and $\gamma$. In Figure 9 we display a log-log plot of $1-F(t)$ against $t$ for large $t$ (corresponding to the 25,000 longest sticking times). The result is consistent with power law behavior with $\gamma \approx 1$.44. For a standard Lévy walk, $\gamma=3-\alpha$ [16], which is consistent with Figure $9 \mathrm{~b}$ showing that $\alpha \approx 1.4$.

\section{Summary}

We have analyzed the system (1.1) describing the position and orientation of an infinitesimal, self-propelled, prolate spheroid, advected by an array of vortices. This is a non-Hamiltonian, non-volume preserving reversible system which, despite its superficial simplicity, displays a range of interesting dynamical properties. Most significantly, on intermediate and/or long time scales the orbits have properties reminiscent of those of Lévy walks, and thus the system provides a framework for Lévy walking in a deterministic setting. The mechanism for this is the existence of elliptic islands, (small) regions of phase space around neutrally stable periodic orbits in which the motion is ballistic; these islands are "sticky" in the sense that other orbits spend long periods of time close to them, giving the long linear stretches characteristic of Lévy walks. For certain values of the parameters the system can also have attracting periodic orbits, which may even be global attractors, in the sense that all orbits outside the elliptic islands are attracted to them. This, however, only happens on very long time scales, and on intermediate scales the Lévy walk behavior is still observed.

Acknowledgments We thank Or Alus, Rainer Klages and Ed Ott for discussions and suggestions. G.A. is thankful for partial support from The Israel Science Foundation (Grant No. 337/12), and DFG grant 1D-84024. 


\section{References}

[1] G. Ariel, A. Be'er and A. Reynolds. Chaotic model for Lévy walks in swarming bacteria. Phys. Rev. Lett. 118, 228102 (2017).

[2] J. Klafter, M.F. Schlesinger and G. Zumofen. Beyond Brownian Motion. Physics Today 49, 33 (1996).

[3] T. Geisel, J. Nierwetberg and A. Zacherl. Accelerated Diffusion in Josephson Junctions and Related Chaotic Systems. Phys. Rev. Lett. 54, 616 (1985).

[4] T. Geisel, A. Zacher and G. Radons. Generic 1/f noise in chaotic Hamiltonian dynamics. Phys. Rev. Lett. 59, 2503 (1987).

[5] G.M. Zaslavsky, D. Stevens and H. Weitzner. Self-similar transport in incomplete chaos. Phys. Rev. E 48, 1683 (1993).

[6] G.M. Zaslavsky. From Hamiltonian chaos to Maxwell's demon. Chaos 5, 653 (1995).

[7] J.D. Meiss and E. Ott. Markov-tree model of intrinsic transport in Hamiltonian systems. Phys. Rev. Lett. 55, 2741 (1985).

[8] J.D. Meiss and E. Ott. Markov tree model of transport in area-preserving maps. Physica D 20387 (1986);

[9] L. Yu, C. Grebogi and E. Ott. Fractal structure in physical space in the dispersal of particles in fluids. In Nonlinear Structures in Physical Systems, edited by L. Lam and H.C. Morris, p. 223, Springer-Verlag, New York (1990).

[10] J.P. Bouchaud, A. Georges, J. Koplik, A. Provata and S. Redner. Superdiffusion in random velocity fields. Phys. Rev. Lett. 64, 2503 (1990).

[11] I.S. Aranson, M.I. Rabinovich and L.Sh. Tsimring. Anomalous diffusion of particles in regular fields. Phys. Lett. A 151, 523 (1990).

[12] O.N. Mesquita, S. Kane and J.P. Gollub. Transport by capillary waves: Fluctuating Stokes drift. Phys. Rev. A 45, 3700 (1992).

[13] T.H. Solomon, A.T. Lee and M.A. Fogleman. Resonant flights and transient superdiffusion in a time-periodic, two-dimensional flow. Physica D 157, 40 (2001).

[14] P. Yves and P. Manneville. Intermittent transition to turbulence in dissipative dynamical systems. Comm. Math. Phys. 74189 (1980).

[15] A. Brandstater and H.L. Swinney. Strange attractors in weakly turbulent Couette-Taylor flow. Phys. Rev. A 35, 2207 (1987).

[16] V. Zaburdeav, S. Denisov and J. Klafter. Lévy walks. Rev. Mod. Phys. 87, 483 (2015).

[17] T. Colin and Z. Neufeld. Transport and aggregation of self-propelled particles in fluid flows. Phys. Rev. Lett. 99, 078101 (2007). 
[18] G. Ariel, A. Rabani, S. Benisty, J.D. Partridge, R.M. Harshey and A. Be'er. Swarming bacteria migrate by Lévy Walk. Nature Comm. 6, 8396 (2015).

[19] M. Harsoula and G. Contopoulos. Global and local diffusion in the standard map. Phys. Rev. E 97, 022215 (2018).

[20] J.A.G. Roberts and G.R.W. Quispel. Chaos and time-reversal symmetry. Order and chaos in reversible dynamical systems. Phys. Rep. 216, 63 (1992).

[21] A Delshams, S V Gonchenko, V S Gonchenko, J T Lazaro and O Sten'kin. Abundance of attracting, repelling and elliptic periodic orbits in two-dimensional reversible maps. Nonlinearity 261 (2013).

[22] A.S. Gonchenko, S.V. Gonchenko, A.O. Kazakov and D.V. Turaev. On the phenomenon of mixed dynamics in Pikovsky-Topaj system of coupled rotators. Physica D 350 (2017) 45.

[23] O. Alus, S. Fishman and J.D. Meiss. Statistics of the island-around-island hierarchy in Hamiltonian phase space. Phys. Rev. E 90, 062923 (2014).

[24] O. Alus, S. Fishman and J.D. Meiss. Universal exponent for transport in mixed Hamiltonian dynamics. Phys. Rev. E 96, 032204 (2017).

[25] R.I. McLachlan, G.R.W. Quispel, and G.S. Turner. Numerical Integrators that Preserve Symmetries and Reversing Symmetries. SIAM J. Numer. Anal., 35586 (1998).

[26] F. Kang and S. Zai-jiu. Volume-preserving algorithms for source-free dynamical systems. Numer. Math. 71, 451 (1995).

[27] R. Klages, S.S.G. Gallegos, J. Solanpää, M. Sarvilahti, and E. Räsänen. Normal and anomalous diffusion in soft Lorentz gases. Phys. Rev. Let. 122, 064102 (2019). 